\title{
Ein molekulares Tumor-Profiling kann die Therapieentscheidung erleichtern
}

Individualisierte, das heißt auf klinisch relevanten patientenindividuellen Biomarkern beruhende, Therapiestrategien sind das übergeordnete Ziel in der Onkologie. Dabei ist die Umset- orientierten Therapieentscheidung zu gelangen. Dass Patienten von einer solchen Vorgehensweise profitieren können, wurde in einer Pilotstudie gezeigt.

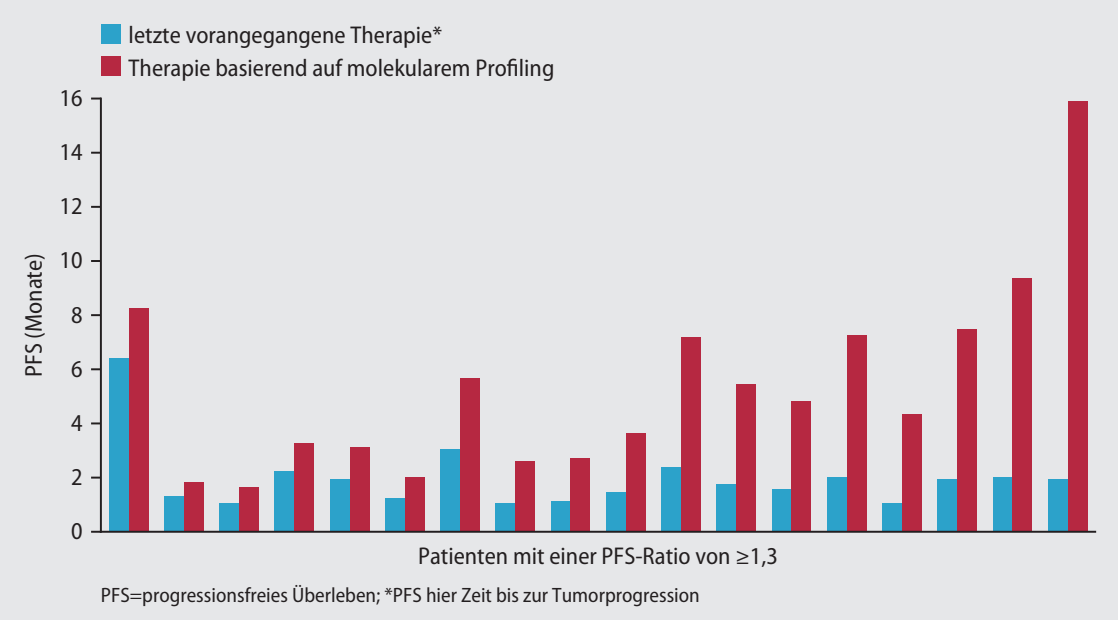

Bisgrove-Studie: progressionsfreies Überleben - aus den Ergebnissen des molekularen Tumor-Profilings abgeleitete Therapie versus letzte vorangegangene Therapie (nach Hoff DD et al., J Clin Oncol 2010, 28:4877-4883)

zung des schnell zunehmenden Wissensstands zu Biomarkern und sogenannten zielgerichteten Therapien im Klinikalltag eine große Herausforderung. Ein spezieller Service für ein molekulares Tumor-Profiling kann jetzt hinzugezogen werden, um zu einer am patientenindividuellen Tumorprofil
Der spezielle Service (Caris Target Now ${ }^{\mathrm{TM}}$ ) beinhaltet eine State-of-the-Art-Biomarker-Analyse des Tumorgewebes sowie einen Report der aktuellen klinischen Evidenz zu spezifischen Therapieoptionen, die sich patientenindividuell aufgrund der im Tumorgewebe identifizierten prädiktiven Biomarker ableiten

\section{Fortgeschrittenes Basalzellkarzinom}

\section{Selektive Blockade mit Vismodegib}

Bei Patienten mit fortgeschrittenem Basalzellkarzinom (BCC) kann durch selektive Blockade des pathologisch aktivierten Hedgehog-Signalwegs eine Regression des Tumors erreicht werden.

In der ERIVANCE-Studie wurde mit dem Hedgehog-Signalweg-Inhibitor Vismodegib bei Patienten mit lokal fortgeschrittenem BCC eine Remissionsrate von $43 \%$ beobachtet, die Rate an Komplettremissionen betrug $21 \%$; bei Patienten mit metastasiertem BCC belief sich die objektive Ansprechrate auf $30 \%$. Damit wurde der primäre Endpunkt erreicht (Sekulic A et al., N Engl J Med 2012, 366:2171-2179). Bis zur beantragten europaweiten Zulassung von Vismodegib bei fortgeschrittenem BCC können Patienten in der globalen STEVIEStudie mit Vismodegib behandelt werden.

nach Informationen der Roche Pharma AG lassen (Ashfaq R, Voss A, European Oncology \& Haematology 2012, 8:242-247). Die Analyse eignet sich dabei für alle soliden Tumoren. Bei Mamma-, Lungen-, Kolorektal- und epithelialen Ovarialkarzinomen können spezifische Profile erstellt werden (www.CarisLifeSciences.eu/ profilemenu). Am häufigsten wird der Tumor-Profiling-Service bei Patienten mit metastasierter bzw. nicht resezierbarer Tumorerkrankung genutzt, wenn beispielsweise mehrere Therapieoptionen verfügbar sind, alle verfügbaren Therapieoptionen ausgereizt wurden oder bei seltenen Tumorerkrankungen keine leitliniengestützte Therapie zur Verfügung steht. Für die Biomarker-Analyse wird Paraffin-eingebettetes Tumorgewebe benötigt, das möglichst von einer erst kürzlich durchgeführten Biopsie oder Tumorresektion stammen sollte.

\section{Klinischer Nutzen des molekularen Tumor-Profilings}

In der prospektiv und multizentrisch durchgeführten Pilotstudie wurde bei 86 Patienten ein molekulares Tumor-Profiling vorgenommen (von Hoff DD et al., J Clin Oncol 2010, 28:4877-4883). Von diesen konnten 66 Patienten eine aus den Ergebnissen des molekularen Tumor-Profilings abgeleitete Therapie erhalten. Darunter waren Patienten mit Mamma$(n=18)$, Kolorektal- $(n=11)$ und Ovarialkarzinom sowie verschiedenen anderen seltenen Tumorerkrankungen $(n=32)$. Alle Patienten waren im metastasierten Stadium und hatten eine Krankheitsprogression auf mindestens zwei vorangegangene Therapien für die fortgeschrittene Tumorerkrankung.

Der klinische Nutzen einer aus den Ergebnissen des molekularen TumorProfilings abgeleiteten Therapie wurde anhand des progressionsfreien Überlebens (PFS) ermittelt; er war definiert als eine PFS-Ratio (PFS unter der aktuellen, aus den Ergebnissen des molekularen Tumor-Profilings abgeleiteten Therapie versus PFS unter der letzten vorangegangenen Therapie) von $\geq 1,3$. Diesen primären Endpunkt der Studie erreichten 18 der 66 Patienten (27\%) (Abb.). Damit konnte die Nullhypothese - eine PFS-Ratio von $\geq 1,3$ bei $15 \%$ der Patienten - verworfen und dieser Ansatz laut den Autoren als vielversprechend gewertet werden.

(am)

nach Informationen von Caris Life Sciences 